\title{
OPTIMAL SYNTHESIS OF EIGHT BAR MECHANISM FOR SPECIFIED PATH GENERATION WITH VARIABLE TOPOLOGY
}

\author{
C. M. Veerendrakumar ${ }^{1}$, Pavan Padara $^{2}$, V. B. Math $^{3}$ \\ ${ }^{1,2,3}$ Research Scholar, Department of Automobile Engineering, Basaveshwar Engineering College, Bagalkot - 587102, \\ Karnataka, India. \\ ${ }^{1}$ Tel: +919448336646; ${ }^{1}$ E-mail: cmveerendra@gmail.com
}

\begin{abstract}
This paper proposes an analytical synthesis method for optimal synthesis of planar eight bar mechanism for path generation with variable topology. The eight bar planar mechanism has been considered to be made of three phases where each of the phases is a four bar mechanism of RRRR type. The method of kinematic synthesis involves the use of Cheybeychev's spacing for locating precision points and Freudenstein's equations for determining link lengths. For any given function, link lengths of the mechanism are determined by assuming the range of input and output angular rotations. Optimized length of rocker link and output angle of the first phase is used as input values for the second phase and same procedure is continued to find the dimensions of the third phase. The combined objective function is established for optimization with transmission angle and link length ratios as design variables. The optimized values of lengths of links of the resulting mechanism satisfy Grashof's condition and have controlled transmission angle. The coupler connected to third phase generates the coupler curve for obtained optimized link lengths. The method developed herein is illustrated with a numerical example.
\end{abstract}

Keywords: Planar Eight Bar Mechanism, Optimal Synthesis, Variable Topology, Path Generation

\section{INTRODUCTION}

Researchers have been inventing a number of different techniques to synthesize planar mechanisms required for machinery. Traditionally graphical synthesis techniques were predominantly used as the use of digital computational facilities was not available to the designers. Analytical design methods used in the design were only those in which complex mathematical computations were not involved. The scenario then changed drastically with the introduction of high speed computers which allowed the Kinematicians to work on new analytical techniques for the design of mechanisms. Use of optimization methods in the synthesis of mechanisms is another interesting development which has improved the design process taking the help of available programming tools. J.A. Cabrera et. al. [1] devised a new method for path synthesis of planar mechanisms. They defined a searching procedure that applies genetic algorithms. The design process is then illustrated by solving problems of synthesis of four bar planar mechanisms. The results obtained by the authors are quite encouraging as the solutions are quite accurate. In a related gradient based optimization was employed by Sancibrian et al. for the path synthesis (R. Sancibrian, F. Viadero, P. Garcia, A. Fernandez, 2004). The design objective is achieved by developing an explicit formulation which resulted in obtaining the values of correct gradient elements. A different notion of orientation structural error was proposed by $\mathrm{H}$. Zhou et. al. for the path synthesis of crank rocker type of planar mechanisms(H. Zhou, Edmund H.M. Cheung, 2001). The difference between the generated path and the desired path is effectively rejected in this method by utilizing this concept of structural error. The procedure avoids making point - wise comparison of the path needed and the actual path created by the designed mechanism. Optimum synthesis is then performed by setting up the mode based upon this error. The global solutions are found using genetic algorithm. A couple of numerical problems are solved to show the efficacy of the new methodology developed.

The trend of using multi - objective genetic algorithms (GAs) in the design of optimal synthesis of mechanisms continued with a reported work of Nariman Zadeh et. al. in which they employed pareto optimum synthesis technique. The method was used for the synthesis of four bar linkages considering the minimization of two objective functions simultaneously (N. Nariman-Zadeh, M. Felezi, A. Jamali, M. Ganji, (2009). Tracking error (TE) and transmission angle's deviation were taken as critical parameters in the analysis part of the design process. The authors have shown that the final design of the mechanism is actually a finding of the middle ground between the two objectives. This is demonstrated by considering the parent fronts into account. The results of the synthesis process are finally compared with the values from other researchers from the literature which are shown to be satisfactory.

Genetic algorithm was further used by Selcuk Erkaya et. al. for checking the influence of the joint clearance on the design of linkages for specified path generation for given transmission angle (Selcuk Erkaya and Ibrahim Uzmay, 2009). The authors assumed joint clearance as a vitural link with no mass. An analytical formulation for the motion of this virtual link was obtained using Lagrange's equation.

Soheil Damangir et al. proposed a new curvature based theory for generating the required path in planar linkages. 
They considered the position vector of a point on coupler link. The path of this point very well matches the desired path and hence is used for directly calculating the function of coupler motion properties (Soheil Damangir et al, 2006).

Francisco T. et. al. applied the approach of combined grossfine optimization technique and computed the value of global optimum in synthesis of planar mechanisms for path generation (Francisco T. Sanchez Marin, and Antonio perez Gonzalez, 2003). The method is innovative in the sense that it employs highly efficient approach by exhaustively reducing the design space where the global optimum is searched.

Irfan Ullah et. al. presented an effective objective function based on Fourier descriptors that evaluates only the shape difference between two curves (Irfan Ullah and Sridar Kota, 1997). The paper proposes a method of minimizing the function utilizing the stochastic global search method.

Bagci, C. et. al. proposed a linear superposition technique in handling a planar synthesis problem of path generation and rigid body guidance with least square formulation (Bagci, $\mathrm{C}$. and Lee, I.P.J, 1975).

The present work involves the use of analytical synthesis techniques for the optimal synthesis of planar eight bar mechanism for path generation with variable topology. The method of kinematic synthesis involves the finding the accuracy points for minimizing he structural error using Cheybeychev's spacing.

The dimensions of the mechanism are then found using Freudenstein's equations. The combined objective function is formulated for optimization by taking the transmission angle and link length ratios as design variables. Grashof's condition is then verified for the designed links of the resulting mechanism.

\section{METHODOLOGY}

\subsection{Synthesis of Planar Eight-Bar Mechanism for}

\section{Path Generation}

The fig-1 shows a planar eight bar mechanism. The mechanism can be treated as operating in three phases. The unknown link lengths are determined by using the Freudenstein's equation.

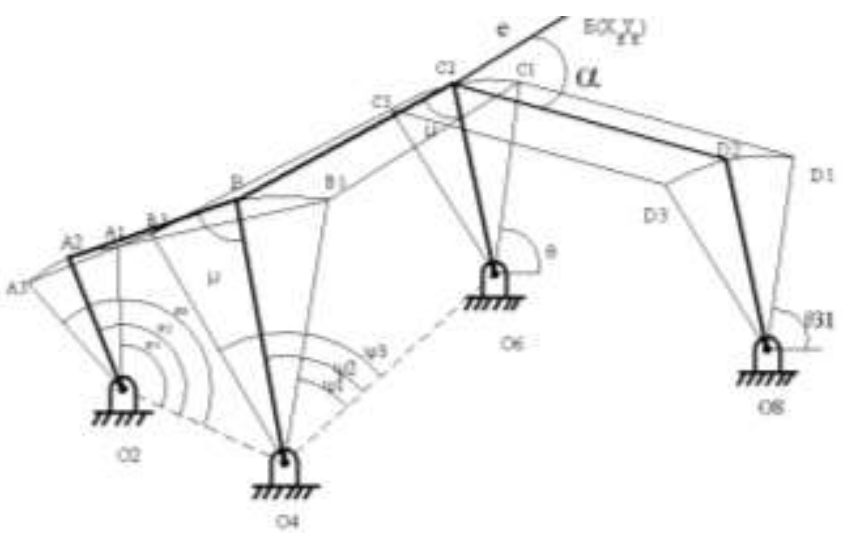

\subsection{Problem Definition}

The problem considered is synthesis of an eight-bar mechanism which generates the given function $y=\log _{10} x$ for which, $1 \leq x \leq 2$.

The eight bar mechanism is considered as a series combinations of three four bar mechanisms. The designing of mechanism for path generation involves the determination of the link lengths of four bar linkage of phase-1 using Freudenstien's equations. This designed mechanism is then tested for compliance to Grashof's condition. Objective function is defined in terms of transmission angles and link length ratios. Optimized link lengths are then obtained with respect to crank angle. Finally the coordinates of tracing point on the coupler of phase- 3 generates the path of tracing point on the coupler of phase-3.

\section{OPTIMIZATION PROCEDURE}

The values of maximum and minimum transmission angles are given by,

$$
\begin{aligned}
& \mu_{\min }=\cos ^{-1}\left[\frac{L_{4}{ }^{2}-\left(L_{1}+L_{2}\right)^{2}+L_{3}{ }^{2}}{2 * L_{4} * L_{3}}\right], \\
& \mu_{\max }=\cos ^{-1}\left[\frac{L_{4}{ }^{2}-\left(L_{1}-L_{2}\right)^{2}+L_{3}{ }^{2}}{2 * L_{4} * L_{3}}\right]
\end{aligned}
$$

The basic objective function is,

$$
U_{1}=\max \left[\left|\frac{\pi}{2}-\mu_{\max }\right| *\left|\frac{\pi}{2}-\mu_{\min }\right|\right]
$$

$\mathrm{U}_{1}$ is used when $\mathrm{L}_{2}$ is very small as compared with the other link lengths. As $L_{2}$ is the shortest link, therefore it is convenient to include the link length ratios as part of the objective function. This function can be written as,

$$
F=\max \left[\frac{L_{1}}{L_{2}}, \frac{L_{3}}{L_{2}}, \frac{L_{4}}{L_{2}}\right]
$$

and,

$$
U_{2}=e^{(F-n)}
$$

Where, ' $n$ ' is an integer that represents the largest acceptable value for the link length ratio. In this work, the typical value for ' $n$ ' is taken as 5 .

The combined objective function is,

$$
U F_{1}=U_{1}+W \times U_{2}
$$

Figure 1. Eight Bar Mechanism 
Where ' $W$ ' is the weighting factor that can be chosen to adjust the relative importance of the length ratio. For good results ' $W$ ' is in between 1 to 5 .

\section{RESULTS AND DISCUSSION}

\section{Phase-1}

Four bar mechanism for the phase-1 includes assumed ground link $\left(\mathrm{L}_{1}\right)$, crank $\left(\mathrm{L}_{2}\right)$, coupler $\left(\mathrm{L}_{3}\right)$, and follower $\left(\mathrm{L}_{4}\right)$. The range of angular displacement of crank and follower are assumed. The values of different parameters are,

$\mathrm{L}_{1}=1$ unit,

Range of the input angle $=0-120^{\circ}$

Range of output angle $=30-150^{\circ}$

$\phi_{1}=22^{0}$

$$
\begin{aligned}
& \psi_{1}=61.22^{0} \\
& \phi_{2}=35^{0} \\
& \psi_{2}=120.19^{0} \\
& \phi_{3}=47.99^{0} \\
& \psi_{3}=164.10^{0}
\end{aligned}
$$

Table-1 consists of designed link lengths and the values of objective function for different values of the input variables. From these values, it is found that objective function is minimum for input angle $0-115^{\circ}$.

Table 1. Computed values of the Link lengths, transmission angles, objective functions (Phase-1)

\begin{tabular}{|l|l|l|l|l|l|l|l|l|}
\hline $\mathbf{I} / \mathbf{p}$ & $\mathbf{O} / \mathbf{p}$ & $\boldsymbol{L}_{\boldsymbol{1}}$ & $\boldsymbol{L}_{\mathbf{2}}$ & $\boldsymbol{L}_{\mathbf{3}}$ & $\boldsymbol{L}_{\boldsymbol{4}}$ & $\boldsymbol{\mu}_{\min }$ & $\boldsymbol{\mu}_{\max }$ & $\boldsymbol{U}$ \\
\hline $0-112$ & $30-150$ & 1 & 4.1214 & 2.0441 & 3.9108 & 52.5903 & 115.1037 & 37.4271 \\
\hline $0-113$ & $30-150$ & 1 & 4.1971 & 2.0429 & 3.9224 & 54.3664 & 117.7085 & 35.6510 \\
\hline $0-114$ & $30-150$ & 1 & 4.2731 & 2.0447 & 3.9362 & 56.1689 & 120.3360 & 33.8480 \\
\hline $\mathbf{0 - 1 1 5}$ & $\mathbf{3 0 - 1 5 0}$ & $\mathbf{1}$ & $\mathbf{4 . 3 5 2 0}$ & $\mathbf{2 . 0 4 9 7}$ & $\mathbf{3 . 9 5 2 4}$ & $\mathbf{5 7 . 9 9 7 2}$ & $\mathbf{1 2 2 . 9 8 5 7}$ & $\mathbf{3 3 . 0 0 2 4}$ \\
\hline $0-116$ & $30-150$ & 1 & 4.4343 & 2.05880 & 3.9709 & 59.8497 & 125.6573 & 35.6738 \\
\hline $0-117$ & $30-150$ & 1 & 4.5202 & 2.0697 & 3.9919 & 61.725 & 128.3506 & 38.3669 \\
\hline $0-118$ & $30-150$ & 1 & 4.6099 & 2.0850 & 4.0154 & 63.6216 & 131.0660 & 41.0821 \\
\hline $0-119$ & $30-150$ & 1 & 4.7039 & 2.1041 & 4.0416 & 65.5384 & 133.8041 & 43.8201 \\
\hline
\end{tabular}

Figure 2 shows the graphical representation of the variation of the objective function with respect to the input angle. It can be seen that the value of the objective function shows a decreasing trend up an input angle of $115^{\circ}$ and then the value increases continuously.

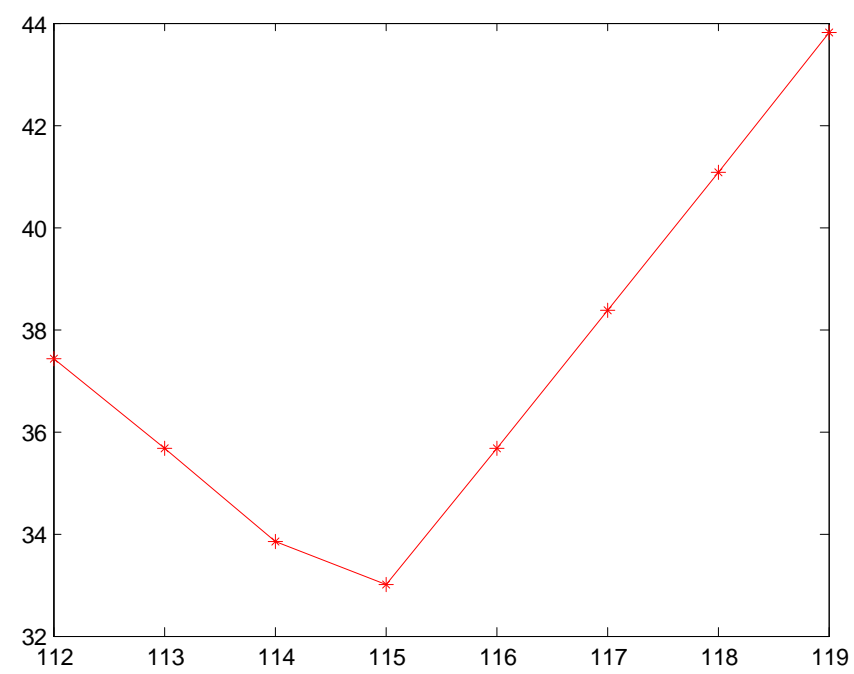

Figure 2. Variation of Objective function with input angle (phase-1)

From above figure 2 it is found that objective function value is minimum at an input angle of $115^{\circ}$. Figure 3 shows the variation of Transmission angle with respect to the input angle.

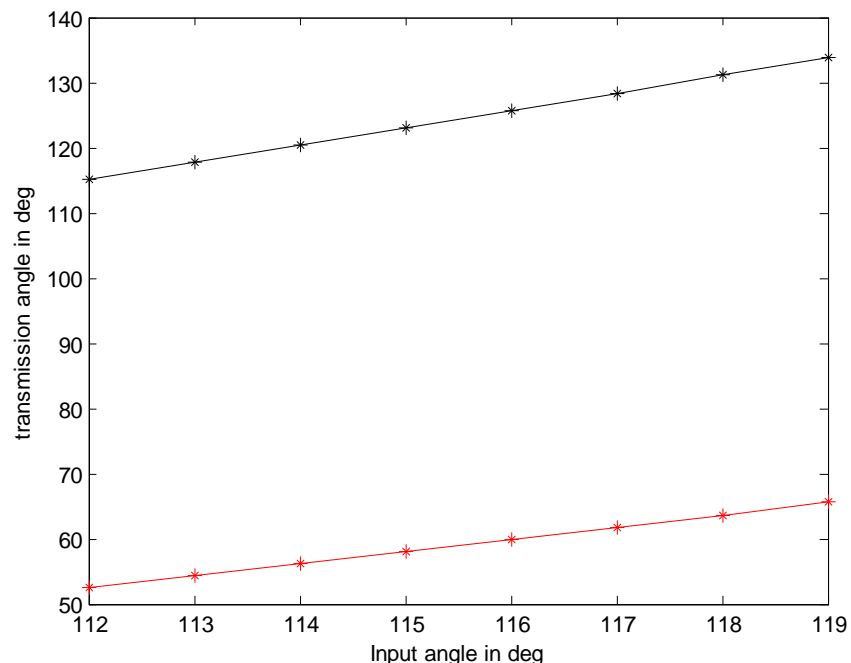

Figure 3. Variation of Transmission angle with Input angle(phase-1)

Linear variation of minimum transmission angle takes place between $52.59^{\circ}$ and $65.54^{\circ}$ and maximum transmission angle between $115.10^{\circ}$ and $133.80^{\circ}$.

\section{Phase-2}

The second phase of the four bar mechanism consists of ground link $\left(\mathrm{O}_{4} \mathrm{O}_{6}\right)$, Crank $\left(\mathrm{L}_{4}\right)$, Coupler $\left(\mathrm{L}_{5}\right)$ and follower $\left(\mathrm{L}_{6}\right)$. In phase-2 length and angle of rotation of the crank $\left(\mathrm{L}_{4}\right.$ $=3.952$ unit) is obtained from the phase- 1 . The values of different parameters are, 

$\phi_{11}=61.225^{0}$
$\psi_{22}=125.098^{0}$
$\psi_{11}=95.622^{0}$
$\phi_{33}=164.102^{0}$
$\phi_{22}=120.195^{0}$
$\psi_{33}=147.051^{0}$

Table-2 Link lengths, transmission angle, objective functions (phase-2)

\begin{tabular}{|l|l|l|l|l|l|l|l|l|l|l|}
\hline$/ \mathrm{p}(1)$ & $\mathrm{O} / \mathrm{p}(1)$ & $\mathrm{I} / \mathrm{p}(2)$ & $\mathrm{O} / \mathrm{p}(2)$ & $L_{4}$ & $L_{5}$ & $L_{6}$ & $L_{7}$ & $\mu_{\min }$ & $\mu_{\max }$ & $U$ \\
\hline $0-115$ & $30-150$ & $30-150$ & $43-126$ & 3.952 & 5.6638 & 5.5506 & 4.8662 & 9.2761 & 103.6894 & 80.7522 \\
\hline $0-115$ & $30-150$ & $30-150$ & $44-128$ & 3.952 & 5.9810 & 5.4054 & 5.3842 & 13.2396 & 110.0620 & 76.7910 \\
\hline $0-115$ & $30-150$ & $30-150$ & $45-130$ & 3.952 & 6.6063 & 5.2823 & 6.1970 & 17.6491 & 116.7970 & 72.3868 \\
\hline $0-115$ & $30-150$ & $30-150$ & $46-132$ & 3.952 & 7.8394 & 5.1813 & 7.6025 & 22.6859 & 123.9015 & 67.3630 \\
\hline $0-115$ & $30-150$ & $30-150$ & $47-134$ & 3.952 & 10.5752 & 5.1031 & 10.5092 & 28.5006 & 131.3723 & 61.5971 \\
\hline $\mathbf{0 - 1 1 5}$ & $\mathbf{3 0 - 1 5 0}$ & $\mathbf{3 0 - 1 5 0}$ & $\mathbf{4 8 - 1 3 6}$ & $\mathbf{3 . 9 5 2}$ & $\mathbf{1 9 . 5 4 0 7}$ & $\mathbf{5 . 0 4 9 0}$ & $\mathbf{1 9 . 6 4 2 0}$ & $\mathbf{3 5 . 2 3 6 6}$ & $\mathbf{1 3 9 . 1 9 5 4}$ & $\mathbf{5 5 . 7 3 3 5}$ \\
\hline $0-115$ & $30-150$ & $30-150$ & $50-140$ & 3.952 & 15.1359 & 5.0245 & 14.7015 & 24.1425 & 127.9926 & 66.1677 \\
\hline $0-115$ & $30-150$ & $30-150$ & $51-142$ & 3.952 & 7.4084 & 5.0635 & 6.7929 & 15.0395 & 117.7545 & 75.0044 \\
\hline
\end{tabular}

From table 5.2 it is observed that objective function is minimum at input angle $30^{\circ}-150^{\circ}$ and output angle $48^{\circ}$ $136^{\circ}$.

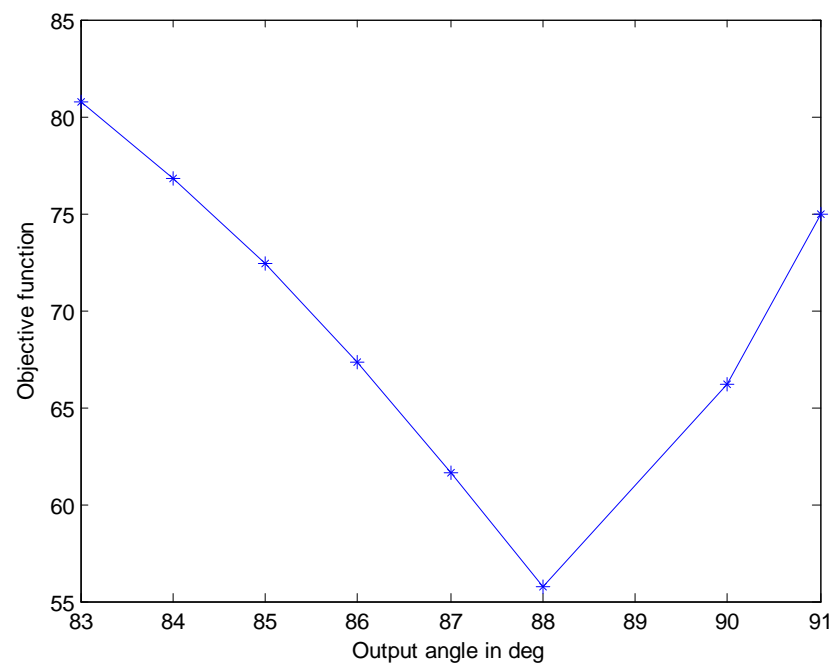

Figure 4. Variation of Objective function with Output angle (phase-2)

\section{Phase-3}

Phase- 3 of the four bar mechanism consists of ground link $\left(\mathrm{O}_{6} \mathrm{O}_{8}\right)$, Crank $\left(\mathrm{L}_{6}\right)$, Coupler $\left(\mathrm{L}_{8}\right)$, follower $\left(\mathrm{L}_{9}\right)$. In phase-3, length and angle of rotation of the crank $\left(\mathrm{L}_{6}=5.05\right.$ units $)$ is obtained from the phase-2. The values of different parameters are,

$$
\begin{aligned}
& \phi_{111}=95.622^{0} \\
& \psi_{111}=42.002^{0} \\
& \phi_{222}=125.098^{0} \\
& \psi_{222}=110.30^{\circ} \\
& \phi_{333}=147.051^{0} \\
& \psi_{333}=161.16^{0}
\end{aligned}
$$

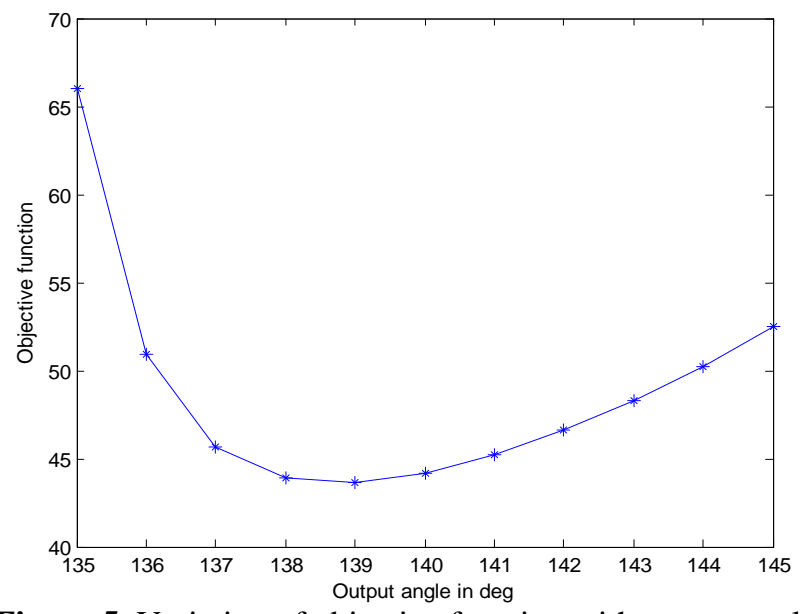

Figure 5. Variation of objective function with output angle (phase-3)

Figure 5 shows the variation of objective function with respect to the output angle. Figure 7 shows the generated optimal coupler curve of the designed mechanism. The values of designed link lengths and the objective function for different values of the input variables are tabulated in Table-3.

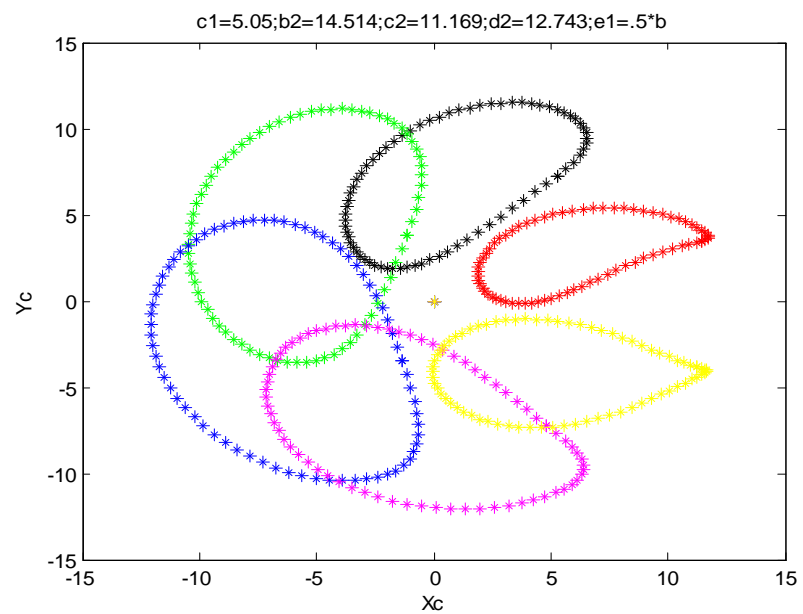

Figure 7. Optimal coupler curve 
Table -3 Link lengths, transmission angle, objective functions for input and output angles(Phase-3)

\begin{tabular}{|c|c|c|c|c|c|c|c|c|c|c|c|c|}
\hline $\begin{array}{c}\mathrm{I} / \mathrm{p} \\
\mathrm{p}-1\end{array}$ & $\begin{array}{c}\mathrm{O} / \mathrm{P} \\
\mathrm{p}-1\end{array}$ & $\begin{array}{c}\mathrm{I} / \mathrm{p} \\
\mathrm{p}-2\end{array}$ & $\begin{array}{c}\mathrm{O} / \mathrm{P} \\
\mathrm{p}-2\end{array}$ & $\begin{array}{c}\mathrm{I} / \mathrm{P} \\
\mathrm{p}-3\end{array}$ & $\begin{array}{c}\mathrm{O} / \mathrm{P} \\
\mathrm{p}-3\end{array}$ & $L_{6}$ & $L_{8}$ & $L_{9}$ & $L_{10}$ & $\mu_{\min }$ & $\mu_{\max }$ & $\mathrm{U}$ \\
\hline $0-115$ & $30-150$ & $30-150$ & $43-126$ & $48-136$ & $25-160$ & 5.05 & 29.39 & 12.97 & 28.25 & 24.57 & 95.77 & 66.39 \\
\hline $0-115$ & $30-150$ & $30-150$ & $44-128$ & $48-136$ & $26-162$ & 5.05 & 22.93 & 12.54 & 21.52 & 39.68 & 92.32 & 50.95 \\
\hline $0-115$ & $30-150$ & $30-150$ & $45-130$ & $48-136$ & $27-164$ & 5.05 & 19.04 & 12.09 & 17.45 & 44.62 & 89.70 & 45.67 \\
\hline $0-115$ & $30-150$ & $30-150$ & $46-132$ & $48-136$ & $28-166$ & 5.05 & 16.42 & 11.63 & 14.71 & 46.31 & 87.85 & 43.86 \\
\hline $\mathbf{0 - 1 1 5}$ & $\mathbf{3 0 - 1 5 0}$ & $\mathbf{3 0 - 1 5 0}$ & $\mathbf{4 7 - 1 3 4}$ & $\mathbf{4 8 - 1 3 6}$ & $\mathbf{2 9 - 1 6 8}$ & $\mathbf{5 . 0 5}$ & $\mathbf{1 4 . 5 1}$ & $\mathbf{1 1 . 1 7}$ & $\mathbf{1 2 . 7 4}$ & $\mathbf{4 6 . 5 1}$ & $\mathbf{8 6 . 6 7}$ & $\mathbf{4 3 . 6 0}$ \\
\hline $0-115$ & $30-150$ & $30-150$ & $48-136$ & $48-136$ & $30-170$ & 5.05 & 13.05 & 10.71 & 11.25 & 45.93 & 86.08 & 44.16 \\
\hline $0-115$ & $30-150$ & $30-150$ & $49-138$ & $48-136$ & $31-172$ & 5.05 & 11.88 & 10.25 & 10.09 & 44.87 & 86.01 & 45.19 \\
\hline $0-115$ & $30-150$ & $30-150$ & $50-140$ & $48-136$ & $32-174$ & 5.05 & 10.91 & 9.79 & 9.15 & 43.47 & 86.42 & 46.58 \\
\hline $0-115$ & $30-150$ & $30-150$ & $51-142$ & $48-136$ & $33-176$ & 5.05 & 10.10 & 9.35 & 8.38 & 41.78 & 87.22 & 48.26 \\
\hline
\end{tabular}

\section{CONCLUSIONS}

A synthesis procedure is developed for the synthesis of planar eight bar mechanism to generate a desired path using variable topology approach. Optimization of the link length is achieved by minimizing the transmission angle. The procedure involves the synthesis in three phases in each phase containing a four bar linkage. Chebychev's spacing is used for locating the precision points and Freudenstein's equations are employed to find the link lengths. The results are then plotted as coupler curves for optimized values of link lengths. The resulting eight bar mechanism satisfies the input and output relation between the crank and the follower motion.

\section{REFERENCES}

[1] Francisco T. Sanchez Marin, and Antonio perez Gonzalez, (2003), "Global optimization in path synthesis based on design space reduction" Mechanism and Machine Theory, Vol.38,pp.579-594.

[2] H. Zhou, Edmund H.M. Cheung, (2001), "Optimal synthesis of crank-rocker linkages for path generation using the orientation structural error of the fixed link", Mechanism and Machine Theory 36, 973-982

[3] Irfan Ullah and Sridar Kota, (1997), "Optimal synthesis of mechanisms for path generation using Fourier descriptors and Global search methods" Transaction of ASME, Vol.119, pp.504-510

[4] J.A. Cabrera, F. Nadal, J.P. Muñoz, A. Simon, (2007), "Multi-objective constrained optimal synthesis of planar Mechanisms using a new evolutionary Algorithm" Mechanism and Machine Theory 42, 791-806

[5] J.A. Cabrera, A. Simon, M. Prado, (2002), "Optimal synthesis of mechanisms with genetic algorithms", Mechanism and Machine Theory 37, 1165-1177

[6] N. Nariman-Zadeh , M. Felezi, A. Jamali, M. Ganji, (2009), "Pareto optimal synthesis of four-bar mechanisms for path generation" Mechanism and Machine Theory ,Vol. 44, pp. 180-191

[7] R. Sancibrian, F. Viadero, P. Garcia, A. Fernandez, (2004), "Gradient-based optimization of path synthesis problems in planar mechanisms" Mechanism and Machine Theory 39 839-856
[8] Robert L. Norton, Design of Machinery, Third edition, Tata McGraw-Hill Publishing Company ltd, New Delhi.

[9] Rudra Pratap, (2008), Getting started with MAT LAB 7, OXFORD University press

[10] Selcuk Erkaya and Ibrahim Uzmay, (2009), "Determining link parameters using genetic algorithms in mechanism with joint clearance" Mechanism and Machine Theory, Vol.44, pp.222-234

[11] Soheil Damangir, Ghazaleh Jafarijashemi, Mohammadhossein Mamduhi, Hassan Zohoor, (2006), "Optimum synthesis of Mechanisms for Path generation using new curvature based Deflection based objective" Proceeding of the $6^{\text {th }}$ WSEAS International Conference on Simulation, Modeling and optimization, Libson, Portugal

[12]Y. Kirani Singh, B.B.Chaudhuri, (2009), MATLAB Programming, PHI learning private Ltd Delhi 